EPJ Web of Conferences 53, 05003 (2013)

DOI: $10.1051 /$ epjconf/20135305003

(C) Owned by the authors, published by EDP Sciences, 2013

\title{
Search for ultra-high energy neutrinos at the pierre auger observatory
}

\author{
S. Navas ${ }^{1, a}$ for the Pierre Auger Collaboration ${ }^{2, b}$ \\ 1 Dpto. de Física Teórica y del Cosmos \& C.A.F.P.E., Universidad de Granada, \\ 18071 Granada, Spain \\ 2 Av. San Martín Norte 304 (5613) Malargüe, Prov. de Mendoza, Argentina
}

\begin{abstract}
The observation of ultra-high energy neutrinos (UHEvs) has become a priority in experimental astroparticle physics. UHEvs can be detected with a variety of techniques. In particular, neutrinos can interact in the atmosphere (downward-going $v$ ) or in the Earth's crust (Earth-skimming $v$ ), producing air showers that can be observed with arrays of detectors at the ground. With the Surface Detector Array of the Pierre Auger Observatory we can detect these types of cascades. The distinguishing signature for neutrino events is the presence of very inclined showers produced close to the ground.In this paper we review the procedure and criteria established to search for UHEvs in the data collected with the surface array of the Pierre Auger Observatory. No neutrino candidates have so far been found, which allows us to place competitive limits to the diffuse flux of UHEvs with energies between $\sim 10^{17} \mathrm{eV}$ and $\sim 10^{20} \mathrm{eV}$. Moreover, upper limits on the neutrino flux from point-like sources have been derived as a function of the source declination. We show that with the Surface Detector of the Pierre Auger Observatory we are sensitive to a large fraction of the sky spanning $\sim 100^{\circ}$ in declination.
\end{abstract}

\section{INTRODUCTION}

The nature and production mechanisms of ultra-high energy cosmic rays (UHECRs), with energies above $10^{18} \mathrm{eV}$, are still unknown [1]. The observation of UHECRs makes an associated flux of ultrahigh energy cosmic neutrinos (UHEvs) [2] very likely. All models of UHECR production predict neutrinos as a result of the decay of charged pions generated in interactions of cosmic rays within the sources themselves ("astrophysical" neutrinos), and/or in their propagation through background radiation fields ("cosmogenic" neutrinos). Also, charged pions, which are photoproduced by UHECR protons interacting with the Cosmic Microwave Background radiation, decay into UHEvs. However, the predicted flux has large uncertainties, since it depends on the UHECR spectrum and on the spatial distribution and cosmological evolution of the sources [2-4]. If UHECRs are heavy nuclei, the UHEv yield is strongly suppressed [5]. The observation of UHE neutrinos would open a new window to the Universe since they travel unaffected by magnetic fields and can give information on astrophysical regions that are otherwise hidden from observation by large amounts of matter.

The Pierre Auger Observatory [6] - located in the province of Mendoza, Argentina, at a mean altitude of $1400 \mathrm{~m}$ above sea level $\left(\sim 875 \mathrm{~g} \mathrm{~cm}^{-2}\right)$ - was designed to measure extensive air showers (EAS) induced by UHECRs. The Fluorescence Detector of the Observatory (FD) [7] comprises a set of imaging telescopes to measure the light emitted by excited atmospheric nitrogen molecules as the EAS develops. A Surface Detector (SD) [8], measures EAS particles at ground with an array of

\footnotetext{
ae-mail: navas@ugr.es

${ }^{b}$ For the full authorlist see Appendix "Collaborations" in this volume

This is an Open Access article distributed under the terms of the Creative Commons Attribution License 2.0, which permits unrestricted use, distribution, and reproduction in any medium, provided the original work is properly cited.
} 
Table 1. Observables and numerical values of cuts applied to select inclined and young showers.

\begin{tabular}{|c|c|c|}
\cline { 2 - 3 } \multicolumn{1}{c|}{} & Earth-skimming $(E S)$ & Downward-going $(D G)$ \\
\cline { 2 - 3 } \multicolumn{1}{c|}{} & Number of Stations $\geq 3$ & Number of Stations $\geq 4$ \\
\hline Inclined & $L / W>5 ; 0.29 \mathrm{~m} \mathrm{~ns}^{-1}<\langle V\rangle<0.31 \mathrm{~m} \mathrm{~ns}^{-1}$ & $L / W>3 ;\langle V\rangle<0.313 \mathrm{~m} \mathrm{~ns}^{-1}$ \\
Showers & $\operatorname{RMS}(V)<0.08 \mathrm{~m} \mathrm{~ns}^{-1}$ & $\operatorname{RMS}(V) /\langle V\rangle<0.08 ; \theta_{\text {rec }}>75^{\circ}$ \\
\hline \hline Young & At least $60 \%$ of stations with & Fisher discriminant $\mathcal{F}$ based \\
Showers & ToT trigger \& AoP $>1.4$ & on Area-over-Peak (AoP) \\
\hline
\end{tabular}

water-Cherenkov detectors ("stations"). Each SD station contains 12 tonnes of water viewed by three 9" photomultipliers. Arranged on a triangular grid with $1.5 \mathrm{~km}$ spacing, 1660 SD stations are deployed over an area of $\sim 3000 \mathrm{~km}^{2}$ which is overlooked by 27 fluorescence telescopes.

Although the primary goal of the SD is to detect UHECRs, it can also identify ultra-high energy neutrinos. Neutrinos of all flavours can interact at any atmospheric depth through charged or neutral currents and induce a "downward-going" $(D G)$ shower. In addition, tau neutrinos can undergo charged current interactions in the Earth's crust and produce a tau lepton which, after emerging from the Earth's surface and decaying in the atmosphere, will induce an "Earth-skimming" $(E S)$ upward-going shower. Even if tau neutrinos are not expected to be produced at the astrophysical source, approximately equal fluxes for each neutrino flavour should reach the Earth as a result of neutrino oscillations over cosmological distances. Neutrino candidate events must be identified against the overwhelming background of showers initiated by standard UHECRs (protons or other nuclei) and, in a much smaller proportion, photons. Highly inclined (zenith angle $\theta>75^{\circ}$ ) $E S$ and $D G$ neutrino-induced showers initiated close to observation level will present a significant electromagnetic component at the ground ("young" showers), producing signals spread over hundreds of nanoseconds in the triggered SD stations. Inclined showers initiated by standard UHECRs are, by contrast, dominated by muons at ground level (“old" showers), with signals typically spread over only tens of nanoseconds.

\section{THE SEARCH FOR UHE NEUTRINOS WITH THE PIERRE AUGER OBSERVATORY}

As stated above, the observation of a significant electromagnetic component (e.m), at ground level, in events with very inclined arrival directions is the key to separate neutrino candidates from nuclear background. Candidates for UHEvs are searched for in inclined showers in the ranges $75^{\circ}<\theta<90^{\circ}$ and $90^{\circ}<\theta<96^{\circ}$ for the $D G$ and $E S$ analyses, respectively. Thanks to the fast sampling (25 ns) of the SD digital electronics (FADC), several observables sensitive to the signal time structure can be used to discriminate between young and old showers, allowing for detection of UHEvs.

Two sets of conditions constituting the $E S$ and $D G$ neutrino identification criteria have been designed and optimized to select showers induced by UHE neutrinos, rejecting those induced by UHECRs (see Table 1). First of all, events occurring during periods of data acquisition instabilities are excluded. The second step in both channels is the selection of inclined showers. From the pattern (footprint) of triggered stations at ground we can extract a length $L$ along the arrival direction of the event (i.e., the main axis of the event) and a width $W$ perpendicular to it characterizing the shape of the footprint [9]. Very inclined events typically have elongated patterns on the ground along the direction of arrival, and hence large values of $L / W$.

Another indication of inclined events is given by the apparent speed of the trigger from a station $i$ to a station $j$, averaged over all pairs $(i, j)$ of stations in the event $(\langle V\rangle)$. Vertical showers have apparent average speeds exceeding the speed of light since all triggers occur at roughly the same time, while in very inclined events $\langle V\rangle$ is concentrated around the speed of light. Moreover its Root-Mean-Square $(\mathrm{RMS}(V))$ is small. Also, and only for downward-going events, a further quality cut is applied consisting on a simple reconstruction of the zenith angle $\theta_{\text {rec }}$ and the requirement that $\theta_{\text {rec }}>75^{\circ}[10]$. 
Table 2. Training and blind search periods for the search for Earth-skimming and downward-going neutrino candidates, together with the equivalent period of time of a full surface detector array, the number of candidates found, the diffuse limits on the flux and the energy range where the limits apply (see text for details).

\begin{tabular}{|c|c|c|}
\hline & Earth-skimming $(E S)$ & Downward-going ( $D G$ \\
\hline Training period & 1 Nov 04-31 Dec 04 & 1 Jan 04-31 Oct 07 \\
\hline Blind search period & 1 Jan 04-31 May 10 & 1 Nov 07-31 May 10 \\
\hline Equivalent full Auger blind search period & $3.5 \mathrm{yr}$ & $2.0 \mathrm{yr}$ \\
\hline$v$ candidates & 0 & 0 \\
\hline Diffuse limit $90 \%$ C.L. $\left(\mathrm{GeV} \mathrm{cm}^{-2} \mathrm{~s}^{-1} \mathrm{sr}^{-1}\right)$ & $k<3.2 \times 10^{-8}$ & $k<1.7 \times 10^{-7}$ \\
\hline Energy range $(\mathrm{EeV})$ & $\sim 0.16-20.0$ & $\sim 0.1-100.0$ \\
\hline
\end{tabular}

Once inclined showers are selected the next step is to identify young showers among the data collected at the SD. A fraction of the data ("training" period) is dedicated to define and optimize the selection algorithm. The remaining fraction ("search" sample) is not used until the selection procedure is established, and then it is "unblinded" to search for neutrino candidates. In Table 2 we indicate the periods used for the training and the "blind" search. The blind search period for the Earth-skimming (downward-going) analysis corresponds to an equivalent of $\sim 3.5 \mathrm{yr}(\sim 2 \mathrm{yr})$ of a full surface detector array consisting of 1660 stations working continuously without interruptions.

In the Earth-skimming analysis we identify young showers by placing a cut on the fraction of stations in the event that fulfill two conditions: (1) the station passes the time over threshold (ToT) local trigger condition, and (2) the ratio of the integrated signal over the peak height - the so-called Area-over-Peak (AoP), a variable that carries information on the time spread of the signal - is greater than 1.4. By convention, both the "area" and the "peak" values are normalized to 1 in signals induced by isolated muons.

In the search for downward-going events, the discrimination power is optimized with the aid of a Fisher discriminant [11]. For that purpose we use variables depending on the AoP of the FADC traces (see [10] for a complete explanation).

In general, the neutrino identification efficiencies depend on many parameters like the energy of the primary neutrino $(D G)$ or tau lepton $(E S)$, the neutrino flavour and type of interaction $(D G)$, the depth in the atmosphere of the $v$ interaction $(D G)$ or the altitude above ground of the tau decay point $(E S)$. The efficiencies are estimated through MC simulations of the first neutrino interaction and the development of the shower in the atmosphere, and from the response of the surface detector array.

\section{THE EXPOSURE TO UHE NEUTRINOS}

For downward-going neutrinos, the calculation of the exposure $\left(\mathcal{E}^{\mathrm{DG}}\right)$ involves folding the SD array aperture with the $v$ interaction probability and the identification efficiency, and integrating in time. Changes in the configuration of the array introduce a dependence of the efficiency $\epsilon$ on the position of the core of the shower $(\mathbf{r})$ in the surface $S$ covered by the array, and on time $t$. Assuming a 1:1:1 flavour ratio the total exposure can be written as [10]:

$$
\mathcal{E}^{\mathrm{DG}}\left(E_{\nu}\right)=\frac{2 \pi}{m} \sum_{i}\left[\sigma^{i}\left(E_{v}\right) \int \sin \theta \cos \theta \epsilon^{i}\left(\mathbf{r}, \theta, D, E_{v}, t\right) d t d \theta d D d S\right]
$$

where the sum runs over the three neutrino flavours and the $\mathrm{CC}$ and $\mathrm{NC}$ interactions, with $\sigma^{i}$ the corresponding $v$-nucleon interaction cross-section [12] and $m$ the nucleon mass. The integral is performed over the zenith angle $\theta$, the interaction depth $D$ of the neutrino (in units of $\mathrm{g} \mathrm{cm}^{-2}$ ), the core positions $\mathbf{r}$ of the showers and the blind search period. For the Earth-skimming neutrinos the calculation of the exposure is described in [9]. 


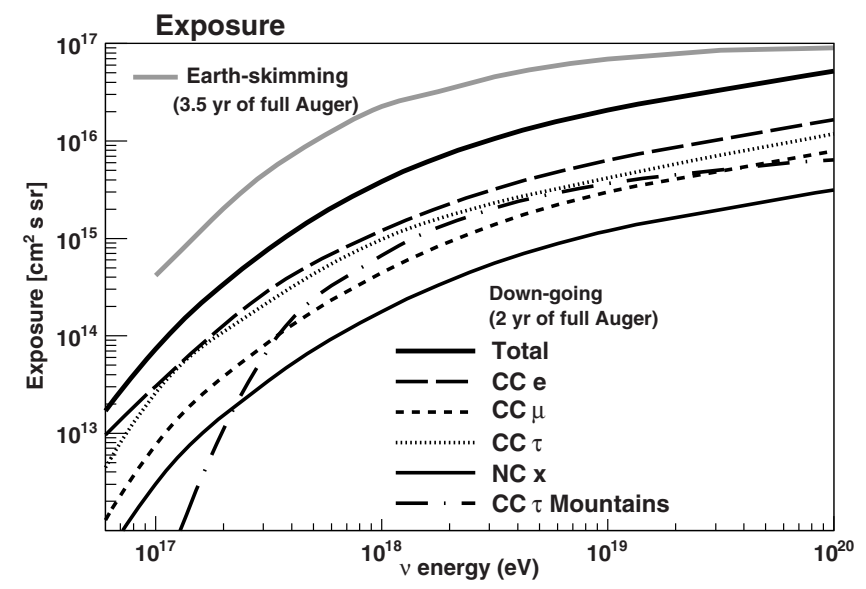

Figure 1. Exposure of the surface detector array of the Pierre Auger Observatory on the data search periods to Earth-skimming $v$-induced showers. and to downward-going $v$-induced showers.

The exposures obtained for the search periods indicated in Table 2 are plotted in Fig. 1. Among the different channels which contribute to the exposure we have included the possibility that downwardgoing $v_{\tau}$ interact with the mountains surrounding the Observatory which provide a dense target for neutrino interactions.

The dominant sources of systematic uncertainties for $D G$ neutrinos come from [10] the hadronic models and the neutrino-induced shower simulations $(+9 \%,-33 \%)$, and from the neutrino interaction cross-section $( \pm 7 \%)$. For the $E S$ channel, they are dominated by the tau energy losses $(+25 \%,-10 \%)$, the shower simulations $(+20 \%,-5 \%)$ and the topography $(+18 \%, 0 \%)$ [9].

\section{LIMITS TO THE DIFFUSE FLUX OF UHE NEUTRINOS}

We have searched for neutrino candidates over the search data periods and no events fulfilling either the Earth-skimming or the downward-going selection cuts were found. Assuming a differential spectrum $\Phi\left(E_{v}\right)=d N_{v} / d E_{v}=k \cdot E_{v}^{-2}$ for the diffuse flux of UHEvs and zero background [9, 10], 90\% C.L. upper limits on the integrated flux of UHE neutrinos reported in Table 2 are derived.

Systematic uncertainties in the exposure were taken into account in the upper limit by using a semi-Bayesian extension [13] of the Feldman-Cousins approach [14]. The limits, shown as two horizontal lines in Figure 2, are valid in the energy ranges $1.6 \times 10^{17} \mathrm{eV} \leq E_{v} \leq 2.0 \times 10^{19} \mathrm{eV}(E S)$ and $1 \times 10^{17} \mathrm{eV}-1 \times 10^{20} \mathrm{eV}(D G)$, where $\approx 90 \%$ of neutrino events would be detected for a $E_{v}^{-2}$ flux. Also shown are the $90 \%$ C.L. upper limits in differential form, where the limits are calculated independently in each energy bin of width 0.5 in $\log _{10} E_{v}$.

The shaded area in Figure 2 brackets the cosmogenic neutrinos fluxes predicted under a wide range of assumptions for the cosmological evolution of the sources, for the transition between the galactic and extragalactic component of cosmic rays, and for the UHECR composition [4]. The corresponding number of cosmogenic neutrino events expected in the blind search sample ranges between 0.1 and 0.3, approximately. For the diffuse flux of cosmogenic neutrinos predicted in [3], 0.6 neutrino events are expected at the Pierre Auger Observatory with the integrated exposure of the present analysis, to be compared with 0.43 events expected in the 333.5 days of live-time of the IceCube-40 neutrino telescope [15]. The current bound to a cosmogenic neutrino flux with energy dependence as in [3] and shown in Figure 2 is 4 times larger than the predicted value. With the current selection criteria the exposure accumulated in $\sim 10$ more years with the Pierre Auger Observatory may exclude this 
UHECR 2012

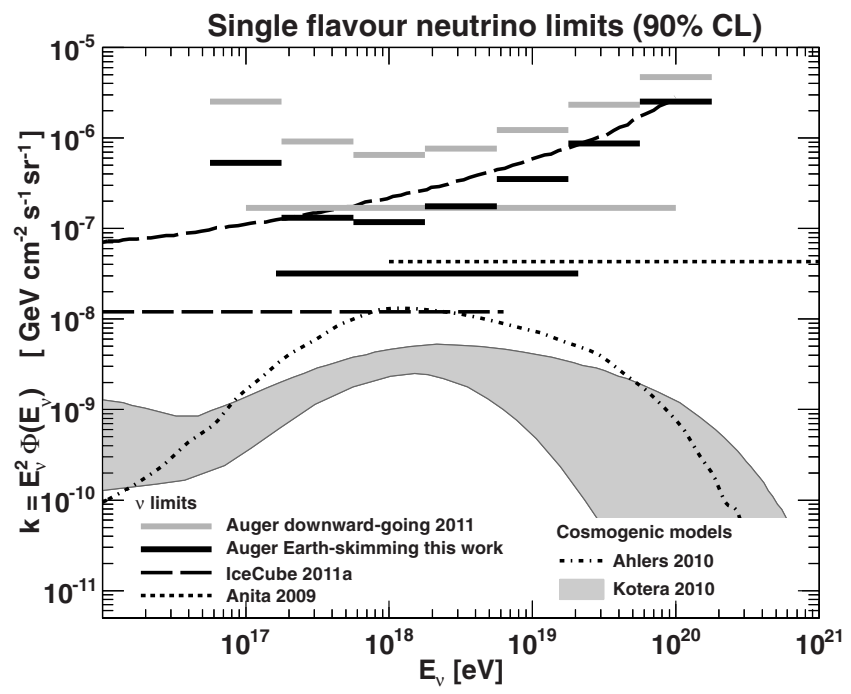

Figure 2. Differential and integrated upper limits at $90 \%$ C.L. on the single flavour $\mathrm{E}_{v}^{-2}$ neutrino flux from the search for downward-going and Earth-skimming neutrinos at the Pierre Auger Observatory. Integrated upper limits are indicated by horizontal lines, with the corresponding differential limits being represented by segments of width 0.5 in $\log _{10} E_{\nu}$. Limits from the IceCube Neutrino Observatory [15] and from the ANITA experiment [16] are also shown after proper rescaling to account for single flavour neutrino flux and different energy binning. Predictions for cosmogenic neutrinos under different assumptions [3,4] are also shown, although predictions almost one order of magnitude lower or higher exist.

cosmogenic neutrino flux at $90 \%$ C.L.. Notice that the maximum sensitivity of the Pierre Auger Observatory, obtained for $E_{v} \sim 10^{18} \mathrm{eV}$, matches well the peak of the expected neutrino flux.

\section{SENSITIVITY TO POINT-LIKE SOURCES}

The neutrino search at the Pierre Auger Observatory is limited to highly inclined showers (zenith angles between $75^{\circ}$ and $96^{\circ}$ ). Thus, at each instant, neutrinos can be detected only from a specific portion of the sky corresponding to these zenith angle ranges. A point-like source of declination $\delta$ and right ascension $\alpha$ (equatorial coordinates) is seen at our latitude $\left(\lambda=-35.2^{\circ}\right)$, at a given sidereal time $t$, with a zenith angle $\theta(t)$ given by:

$$
\cos \theta(t)=\sin \lambda \sin \delta+\cos \lambda \cos \delta \sin (2 \pi t / T-\alpha),
$$

where $T$ is the duration of one sidereal day. From equation 2, the fraction of a sidereal day during which a source is detectable (i.e., within the zenith angle ranges for the $E S$ and $D G$ analyses) depends only on the source declination. The SD of the Pierre Auger Observatory is sensitive to point-like sources of neutrinos over a broad declination range spanning north of $\delta \sim-65^{\circ}$ and south of $\delta \sim 55^{\circ}$. The regions of the sky close to the Northern $\left(\delta=90^{\circ}\right)$ and Southern $\left(\delta=-90^{\circ}\right)$ Terrestrial Poles are not accessible by this analysis. As an example, Centaurus A $\left(\delta \sim-43^{\circ}\right)$ is observed $\sim 7 \%(\sim 15 \%)$ of one sidereal day in the range of zenith angles corresponding to the $E S(D G)$ search.

The exposure of the SD as a function of the neutrino energy and of the source position in the sky, $\mathcal{E}^{P S}\left(E_{v}, \delta\right)$, is evaluated by folding the SD aperture with the neutrino interaction probability and the selection efficiency for each neutrino channel. The procedure is identical to that used for the calculation of the exposure for a diffuse flux of UHEvs described in Section 3, with the exception of the solid angle integration over the sky. 


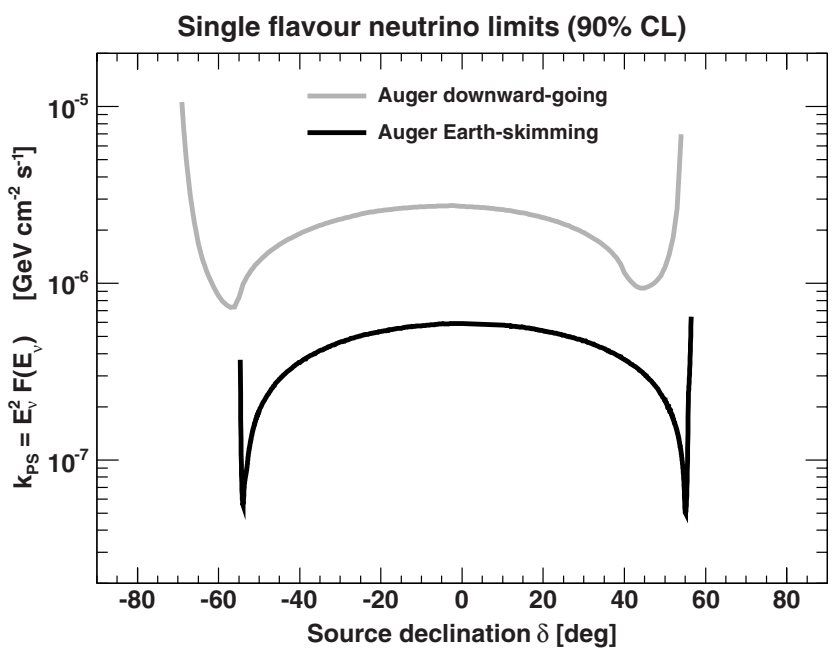

Figure 3. Upper limits at $90 \%$ C.L. on a single flavour $E_{v}^{-2}$ flux from a specific point-like source as a function of the source declination [17]. The bounds from the Earth-skimming and downward-going neutrino analyses hold for a neutrino energy range $10^{17}-10^{20} \mathrm{eV}$ (see text for details).

To set the upper limit, a differential flux $F\left(E_{v}\right)=k_{P S}(\delta) \cdot E_{v}^{-2}$ was assumed, as well as a 1:1:1 neutrino flavour ratio. Systematic uncertainties on the exposure were calculated using the semi-Bayesian approach described above in Section 4. In Figure 3, the 90\% C.L. upper limits on $k_{P S}$ derived from the $E S$ and $D G$ analyses are shown as a function of source declination. Limits for $k_{P S}$ at the level of $\approx 5 \times 10^{-7}$ and $2.5 \times 10^{-6} \mathrm{GeV} \mathrm{cm}^{-2} \mathrm{~s}^{-1}$ were obtained over a broad range of declinations from the searches of Earth-skimming and downward-going neutrinos, respectively. The shape of the declinationdependent upper limits is largely determined by the fraction of time a source is within the field of view of the $E S$ or $D G$ analyses, and, to a lesser extent, by the zenith angle dependence of the exposure. The upper limits are derived for neutrinos in the energy range $1.6 \times 10^{17} \mathrm{eV}-2.0 \times 10^{19} \mathrm{eV}$ for the Earth-skimming analysis, and in the energy range $1 \times 10^{17} \mathrm{eV}-1 \times 10^{20} \mathrm{eV}$ for the downward-going analysis, with a negligible dependence of these energy intervals on the source declination. These are the best limits around $1 \mathrm{EeV}$.

For the particular case of the active galaxy Centaurus A, a potential source of UHECRs, the expected number of events in our blind search samples for a flux like in [18] is about 0.1 and 0.02 for the $E S$ and $D G$ selection respectively, the expected number for [19] being one order of magnitude smaller.

Special thanks to the organisers of the conference for inviting me to present these results on behalf of the Pierre Auger Collaboration and to all my colleagues from Auger for their remarkable efforts on improving the quality of our results. I also acknowledge support from Spanish Ministerio de Ciencia e Innovación grants FPA2009-07187 and AIC-B-2011-0700.

\section{References}

[1] F. Halzen \& D. Hooper, Rep. Prog. Phys. 65, 2002, 1025

[2] J.K. Becker, Phys. Rep. 458, 2008, 173

[3] M. Ahlers et al., Astropart. Phys. 34, 2010, 106 


\section{UHECR 2012}

[4] K. Kotera et al., JCAP 10, 2010, 013

[5] M. Ave et al., Astropart. Phys. 23, 2005, 19

[6] The Pierre Auger Collaboration, Nucl. Instr. and Meth. A 523, 2004, 50

[7] The Pierre Auger Collaboration, Nucl. Instr. and Meth. A 620, 2010, 227

[8] I. Allekotte et al., Nucl. Instr. and Meth. A 586, 2008, 409

[9] The Pierre Auger Collaboration, Phys. Rev. D 79, 2009, 102001

[10] The Pierre Auger Collaboration, Phys. Rev. D 84, 2011, 122005

[11] R. Fisher, Ann. Eugenics 7, 1936, 179

[12] A. Cooper-Sarkar and S. Sarkar, JHEP 01, 2008, 075

[13] J. Conrad et al., Phys. Rev. D 67, 2003, 12002

[14] G. J. Feldman and R. D. Cousins, Phys. Rev. D 57, 1998, 3873

[15] The IceCube Collaboration, Phys. Rev. D 83, 2011, 092003

[16] The ANITA Collaboration, Phys. Rev. D 82, 2010, 022004. Erratum arXiv:1011.5004v1 [astro-ph]

[17] The Pierre Auger Collaboration, Astrophys. J. Lett., 2012 (accepted for publication)

[18] A. Cuoco \& S. Hannestad, Phys. Rev. D 78, 2008, 023007

[19] M. Kachelriess et al., New J. Phys. 11, 2009, 065017 